American Journal of Applied Sciences 6 (12): 2030-2035, 2009

ISSN 1546-9239

(C) 2009 Science Publications

\title{
Synthesis and Characterization of Silver/Clay/Chitosan Bionanocomposites by UV-Irradiation Method
}

\author{
${ }^{1}$ Mansor Bin Ahmad, ${ }^{1}$ Kamyar Shameli, ${ }^{2}$ Majid Darroudi, \\ ${ }^{1}$ Wan Md Zin Wan Yunus and ${ }^{1}$ Nor Azowa Ibrahim \\ ${ }^{1}$ Department of Chemistry, Faculty of Science, University Putra Malaysia, \\ 43400 UPM Serdang, Selangor, Malaysia \\ ${ }^{2}$ Advanced Materials and Nanotechnology Laboratory, Institute of Advanced Technology, \\ University Putra Malaysia, 43400 UPM Serdang, Selangor, Malaysia
}

\begin{abstract}
Problem statement: Silver/Montmorillonite/Chitosan Bionanocomposites (Ag/MMT/Cts BNCs) have been synthesized by UV-irradiation reduction method in the absence of any reducing agent or heat treatment which is used to antibacterial application and medical devices. Approach: MMT, Chitosan and $\mathrm{AgNO}_{3}$ were used as a solid support, stabilizer and silver precursor, respectively. The properties of $\mathrm{Ag} / \mathrm{MMT} / \mathrm{Cts} \mathrm{BNCs}$ were studied as a function of UV-irradiation times. The crystalline structure, d-spacing of interlayer of MMT, the size distributions and surface plasmon resonance of synthesized silver nanoparticles (Ag-NPs) were characterized using Powder X-Ray Diffraction (PXRD), Transmission Electron Microscopy (TEM) and UV-vis spectroscopy. The functional groups of prepared BNCs were also determined by Fourier Transform Infrared (FT-IR). Results: The results obtained from UV-vis spectroscopy of synthesized Ag-NPs showed that the intensity of the maximum wavelength of the plasmon peaks were increased with the increasing in the UV-irradiation times. Results from UV-visible spectroscopy and Transmission Electron Microscopy (TEM) microphotographs show that particles size of Ag-NPs decrease with the increase of UV-irradiation time. Conclusion: UV-irradiation disintegrated the Ag-NPs into smaller size until a relatively stable size and size distribution were achieved. Ag/MMT/Cts BNCs could be suitable to antimicrobial applications and medical devices.
\end{abstract}

Key words: Silver Nanoparticles, Bionanocomposites, UV-irradiation, powder X-ray diffraction

\section{INTRODUCTION}

Due to their excellent properties, metal/clay/polymer compounds as Bionanocomposites (BNCs) have become a promising new research field. $\mathrm{BNCs}$ are made of natural polymer matrix and organic/inorganic filler with at least one dimension on the nanometer scale. Among natural polymers chitosan has been extensively investigated as a natural cationic biopolymer with excellent bioactivity, biodegradability, biocompatibility, nontoxicity and multi functional groups as well as solubility in aqueous medium for food packaging film, bone substitutes and artificial $\mathrm{skin}^{[1-4]}$.

MMT as a lamellar clay has intercalation, swelling and ion exchange properties. Its interlayer space has been used for the synthesis of material and biomaterial nanoparticles, as support for anchoring transition-metal complex catalysts and as adsorbents for cationic ions ${ }^{5-}$ 7]. Ag-NPs possess many interesting and unique properties. It is found in various applications, such as catalysis, electronics, non-linear optics, antimicrobial and biomaterial applications ${ }^{[8]}$. Several methods have been reported for the synthesis of Ag-NPs, e.g., photochemical reduction ${ }^{[9]}$, microwave ${ }^{[10]}$, chemical reduction $^{[11]}, \gamma$-irradiation ${ }^{[12]}$. In this study, we report the synthesis of $\mathrm{Ag} / \mathrm{MMT} / \mathrm{Cts} \mathrm{BNCs}$ by UV-irradiation method at different irradiation times.

\section{MATERIALS AND METHODS}

Materials: All reagents were of analytical grades and were used as received without further purification. $\mathrm{AgNO}_{3}$ (99.98\%), used as silver precursor, was supplied from Merck, Germany. MMT, used as a solid support for Ag-NPs, was purchased from Kunipa-F, Japan. Chitosan (Low molecular weight, SigmaAldrich, USA) was used as a stabilizer agent. Glacial acetic acid (HAC, 99\%), as a solvent for chitosan, was

Corresponding Author: Mansor Bin Ahmad, Department of Chemistry, Faculty of Science, Universiti Putra Malaysia, 43400 UPM Serdang, Selangor, Malaysia 
purchased from Sigma-Aldrich, USA. All aqueous solutions were prepared with Double Distilled water (DD-water).

Synthesis of Ag/MMT/Cts BNCs: Chitosan sol $(100 \mathrm{~mL}, 2.0 \mathrm{wt} \%)$ were prepared by solubilizing chitosan in $1.0 \mathrm{wt} \%$ of HAC sol $(\mathrm{pH} 3.45)$ under constant stirring for $1 \mathrm{~h}$. Five hundred $\mathrm{ml}$ of $\mathrm{AgNO}_{3}$ $(0.02 \mathrm{M})$ was added into the chitosan sol under constant stirring for preparation of $\mathrm{Cts} / \mathrm{AgNO}_{3}$ sol. For preparation of MMT suspension, 5.0 g of MMT was dispersed in $400 \mathrm{~mL}$ DD-water and was vigorously stirred for $1 \mathrm{~h}$. The Cts/ $/ \mathrm{gNO}_{3}$ was added into the MMT suspension and the mixture was further vigorously stirred for $4 \mathrm{~h}$ at the room temperature to obtained MMT/Cts/ $/ \mathrm{AgNO}_{3}$ sol. The MMT/Cts/ $/ \mathrm{AgNO}_{3}$ sol was irradiated using the UV reactor with UV-lamp at $\lambda=365 \mathrm{~nm}$ while it was stirred at speed of $195 \mathrm{rpm}$. The irradiation times of $1 \mathrm{~h}$ (S1), $3 \mathrm{~h}$ (S2), $18 \mathrm{~h}$ (S3), $48 \mathrm{~h}$ (S4) and $96 \mathrm{~h}$ (S5) were applied for different cuvettes, respectively. Then, obtained suspensions of $\mathrm{Ag} / \mathrm{MMT} / \mathrm{Cts} \mathrm{BNCs}$ were centrifuged, washed with DD-water twice and dried at $40^{\circ} \mathrm{C}$ under vacuum overnight. All experiments were conducted at ambient temperature.

Characterization methods and instrumentation: The Ag/MMT/Cts BNCs were characterized using ultraviolet-visible (UV-vis) spectroscopy, Transmission Electron Microscopy (TEM), powder Xray diffraction (PXRD) and Fourier Transform infrared spectroscopy (FT-IR). The UV-vis spectra were recorded over the range of $300-700 \mathrm{~nm}$ with (H.UV.1650 PC, SHIMADZU) UV-vis spectrophotometer. Transmission electron microscopy (TEM) observations were carried out on A Hitachi H7100 electron microscope and the particle size distributions were determined using the UTHSCSA Image Tool version 3.00 program. The structure of $\mathrm{Ag} / \mathrm{MMT} / \mathrm{Cts} \mathrm{BNCs}$ has been studied using Powder X-Ray Diffraction (PXRD-6000 SHIMADZU). The change in interlamellar spacing of MMT and $\mathrm{Ag} / \mathrm{MMT} / \mathrm{Cts}$ BNCs was also studied by PXRD in the angle range of $2^{\circ}<2 \theta<12^{\circ}$. The interlamellar space was calculated from the PXRD peak positions using Bragg's law. A wavelength $(\lambda)$ equal to $0.15418 \mathrm{~nm}$ was used for these measurements. The PXRD patterns were recorded at a scan speed of $2^{\circ} \mathrm{min}^{-1}$. FT-IR spectra were recorded over the range of $\left(400-4000 \mathrm{~cm}^{-1}\right)$ with a series 100 Perkin Elmer FT-IR 1650 spectrophotometer, The reactions were carried out on a UV reactor (UV-A, 6W).

\section{RESULTS}

TEM images and their size distributions of AgNPs show the mean diameter of the nanoparticles mostly ranged from 3-5 nm (Fig. 1). The comparison between PXRD patterns of MMT and $\mathrm{Ag} / \mathrm{MMT} / \mathrm{Cts}$ BNCs in the small angle range of $2 \theta\left(2^{\circ}<2 \theta<12^{\circ}\right)$, indicate the formation of the intercalated structure (Fig. 2). The PXRD pattern was also employed to determine the crystalline structure of the synthesized Ag-NPs (Fig. 3). The formation of Ag-NPs was also followed by measuring the surface plasmon resonance (SPR) of the MMT/Cts suspensions containing AgNPs at the wavelength ranged from 300-700 nm (Fig. 4). The chemical structure of the MMT/Cts and $\mathrm{Ag} / \mathrm{MMT} / \mathrm{Cts}$ BNCs were analyzed by using FT-IR spectroscopy (Fig. 5 and 6).

\section{DISCUSSION}

The color of prepared samples at different UVirradiation times gradually changed from colorless to light gray, then to gray and finally to dark gray, indicating the formation of Ag-NPs in MMT/Cts suspension. When the MMT/Cts/ $\mathrm{AgNO}_{3}$ suspension was irradiated under UV for $3 \mathrm{~h}$ (S2), photo-reduced Ag-NPs were formed with a broad size distribution and mean diameter of about $4.60 \pm 1.31 \mathrm{~nm}$ (Fig. 1a and b). As it can be seen from (Fig. 1c and d), when the irradiation time was increased in $\mathrm{S} 4$, the mean diameter of Ag-NPs decreased considerably to $3.16 \pm 0.95 \mathrm{~nm}$ as compared to S2. It can be seen that larger Ag-NPs were obtained under shorter irradiation time and they disintegrated under the further UVirradiation $^{[13]}$.

Photo-induced fragmentation of Ag-NPs has been reported by Kamat et al. ${ }^{[14]}$. Briefly described as the following:

$(\mathrm{Ag})_{\mathrm{n}}+2 \mathrm{~h} v \rightarrow(\mathrm{Ag})_{\mathrm{n}}^{+}+\mathrm{e}_{\mathrm{aq}}^{-}$

$(\mathrm{Ag})_{\mathrm{n}}^{+}+\mathrm{e}_{\text {aq }}^{-} \rightarrow(\mathrm{Ag})_{\mathrm{n}}$

$(\mathrm{Ag})_{\mathrm{n}}^{+} \rightarrow(\mathrm{Ag})_{\mathrm{n}-1}+\mathrm{Ag}^{+}$

Where:

$(\mathrm{Ag})_{\mathrm{n}}=$ The silver nanocluster containing $(\mathrm{n})$ silver atom

$\mathrm{e}_{\mathrm{aq}}^{-} \quad=$ The aqueous electron 

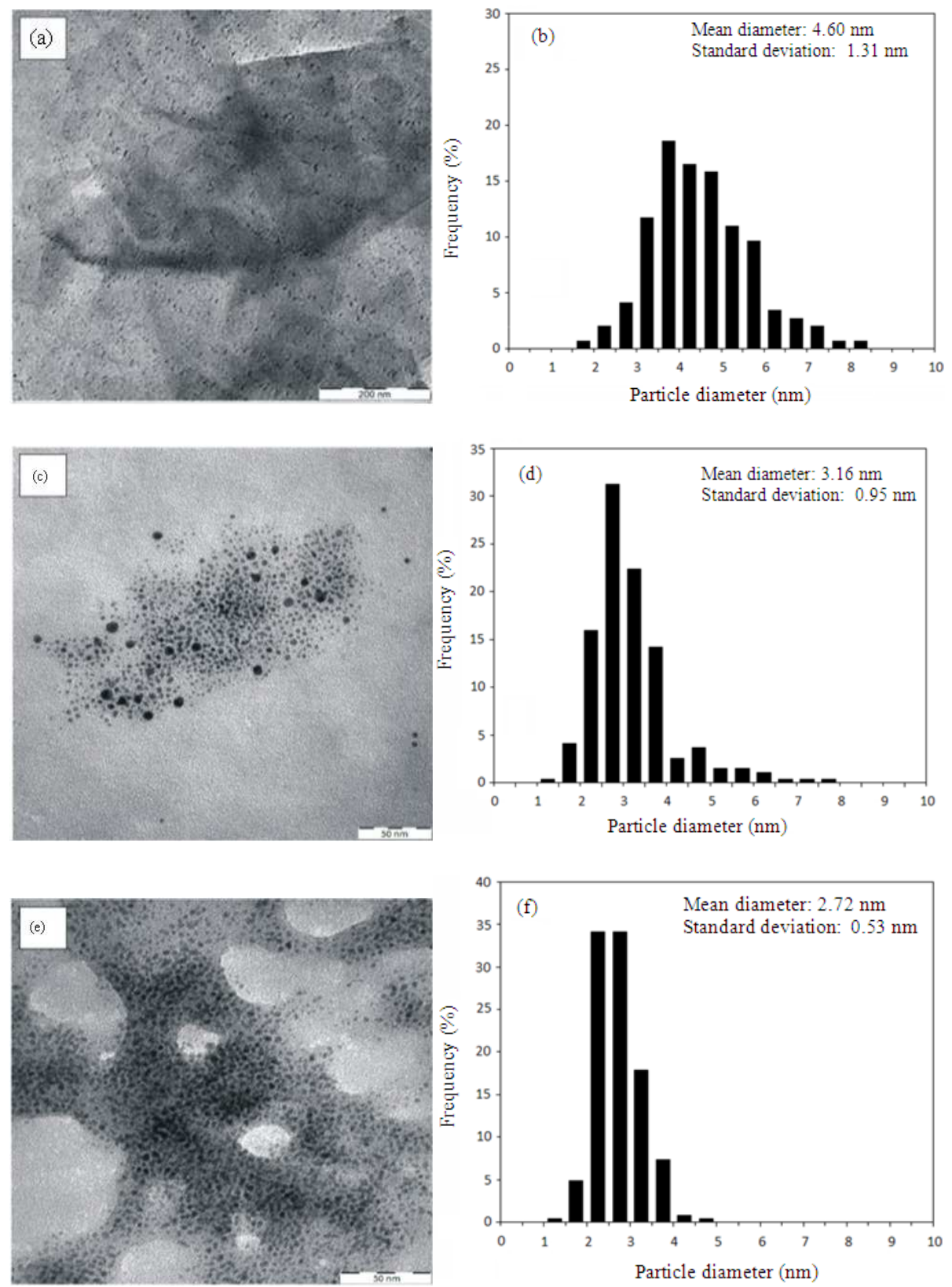

Fig. 1: TEM images and their corresponding particle size distributions of Ag/MMT/Cts BNCs for S2 (a and b), S4 (c and d) and S5 (e and f)

After UV-irradiation on aqueous suspensions of $\mathrm{MMT} / \mathrm{Cts} / \mathrm{AgNO}_{3}$, a large amount of aqueous electrons $\mathrm{e}_{\mathrm{aq}}^{-}$was produced and the $\mathrm{Ag}^{+}$ions were reduced into Ag. The d-spacing $\left(d_{L}\right)$ between layers of MMT was occupied by elemental $\mathrm{Ag}$ atoms. Then, there is a higher possibility for the single $\mathrm{Ag}$ atoms to nucleate and grow into $(\mathrm{Ag})_{\mathrm{n}}$ clusters rather than being oxidized into $\mathrm{Ag}^{+}$ions again because the $(\mathrm{Ag})_{\mathrm{n}}$ clusters are protected by the MMT/Cts due to their special structure. As shown in Fig. 1e and f, no large decrease in the particle size and change in size distribution can be observed in S5 $(2.72 \pm 0.53 \mathrm{~nm})$, when compared with S4. This indicates that the Ag-NPs obtained in this irradiation time are extremely stable.

As shown in Fig. 2, the original $\mathrm{d}_{\mathrm{L}}$ of MMT, $1.24 \mathrm{~nm}$ and in MMT/Cts is increased to1.67 nm at 
smaller $2 \theta$ angles $\left(2 \theta=7.12^{\circ}\right.$ for MMT and $2 \theta=5.26^{\circ}$ for MMT/Cts) by chitosan intercalation. The $\mathrm{d}_{\mathrm{L}}$ in $\mathrm{S} 5$ also was increase to $1.70 \mathrm{~nm}$ at smaller $2 \theta$ angles $\left(2 \theta=5.16^{\circ}\right)$ by silver intercalation. Theses $d_{L}$ values are direct proof of intercalation structures. Metallic nanoparticles formed at the latter location are the cause of the increase in basal spacing. In these samples the intensities of the reflections are significantly lower whereas their half-widths are larger than those of undoped clay minerals: the highly ordered parallel lamellar structure of the mineral is disrupted by particle formation $^{[15]}$.

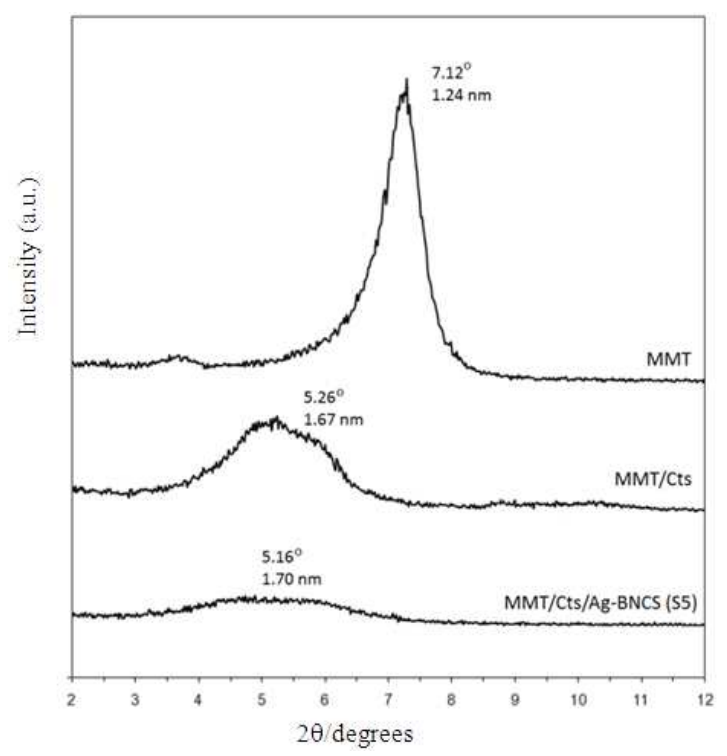

Fig. 2: PXRD patterns of MMT, MMT/Cts and Ag/MMT/Cts BNCs (S5)

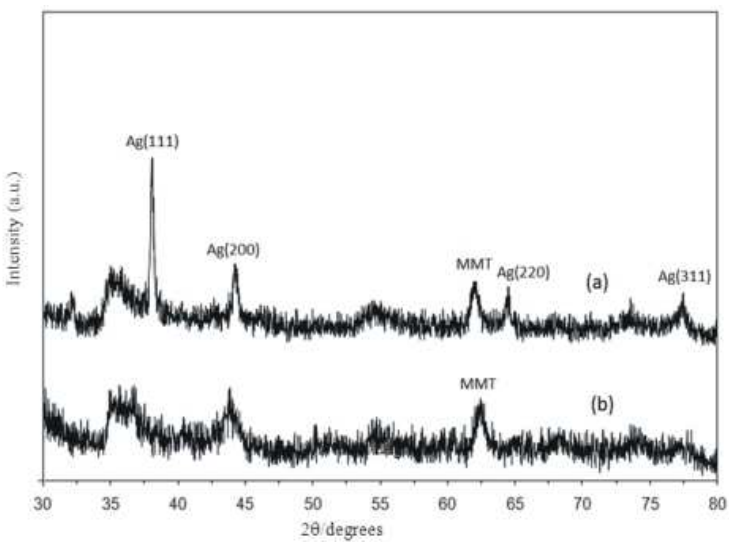

Fig. 3: PXRD patterns of MMT and Ag/MMT/Cts BNCs (S5) (a) MMt (b) MMT/Cts/Ag-BNCs (S5)
The PXRD peaks at $2 \theta$ of $38.07,44.23,64.52$ and $77.30^{\circ}$ (Fig. 3) can be attributed to the (111), (200) (220) and (311) crystallographic planes of face-centered cubic (fcc) silver crystals, respectively ${ }^{[16]}$. The PXRD peak broadenings of Ag-NPs are mostly because of existing of nano-sized particle ${ }^{[17]}$. In addition there is a characteristic peak at about $2 \theta=62.5^{\circ}$ that related to MMT clay (PXRD Ref. No. 00-003-0010) as a stable substrate.

The characteristic silver Surface Plasmon Resonance (SPR) band was detected around $330 \mathrm{~nm}$ (Fig. 4), when the UV-irradiation duration exceeded 3 h. For S3 and S4 the absorbance was observed at 331 $\mathrm{nm}$ and its intensity obviously has increased compared to S2. The increase of the absorbance with further UVIrradiation time indicates that the concentration of $\mathrm{Ag}$ NPsincreases ${ }^{[18]}$ because the photo-induced fragmentation of Ag-NPs has increased the total number of particles in the solution. For S5, the absorbance increased considerably and was red-shifted to $336 \mathrm{~nm}$. This is consistent with earlier findings that at higher concentration of metal nanoparticles may also lead to the red-shift of SPR band ${ }^{[19]}$.

The FT-IR spectrum of MMT (Fig. 5c) shows the vibration bands at $3619 \mathrm{~cm}^{-1}$ for $\mathrm{O}-\mathrm{H}$ stretching, $3425 \mathrm{~cm}^{-1}$ due to interlayered $\mathrm{O}-\mathrm{H}$ stretching $(\mathrm{H}-$ bonding), at the 1637 and $1502 \mathrm{~cm}^{-1}$ for $\mathrm{H}-\mathrm{O}-\mathrm{H}$ bending, 984 and $896 \mathrm{~cm}^{-1}$ for Si-O stretching, $594 \mathrm{~cm}^{-1}$ for $\mathrm{Al}-\mathrm{OH}, 896 \mathrm{~cm}^{-1}$ due to $(\mathrm{Al}, \mathrm{Mg})-\mathrm{OH}$ vibration modes and 518 and $430 \mathrm{~cm}^{-1}$ for Si-O bending ${ }^{[20]}$.

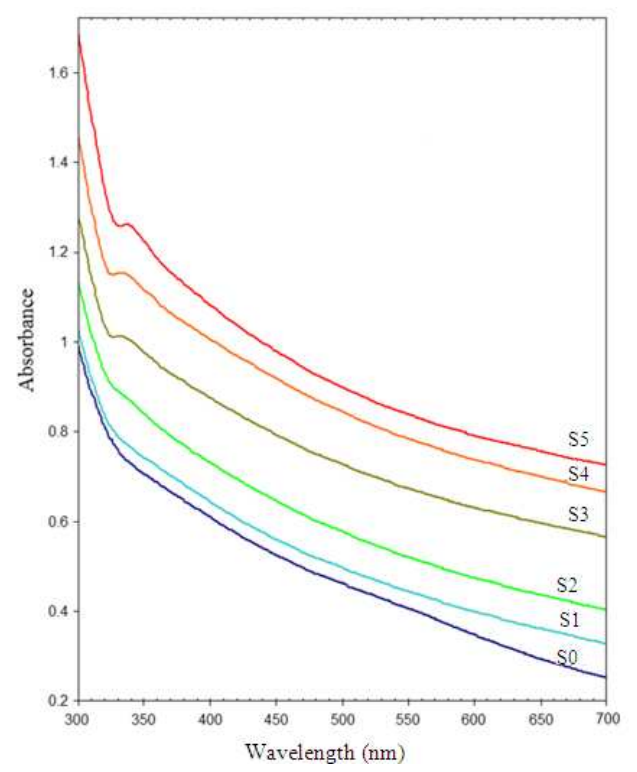

Fig. 4: UV-vis adsorption spectra of $\mathrm{Ag} / \mathrm{MMT} / \mathrm{Cts}$ BNCs at different irradiation times 


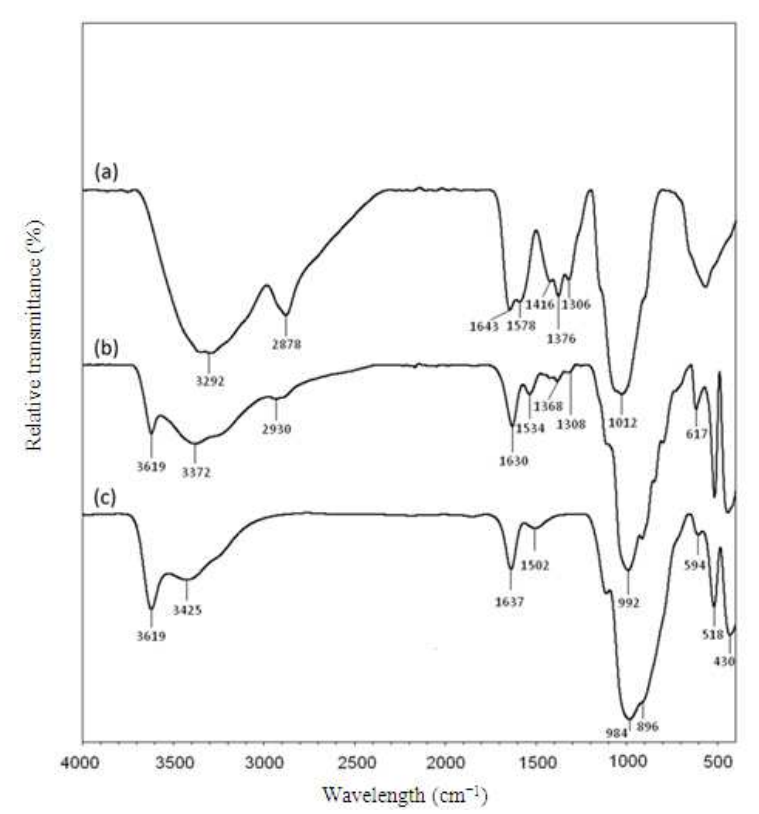

Fig. 5: FT-IR spectra of MMT, Cts and MMT/Cts (a) Cts (b) MMT/Cts (c) MMT

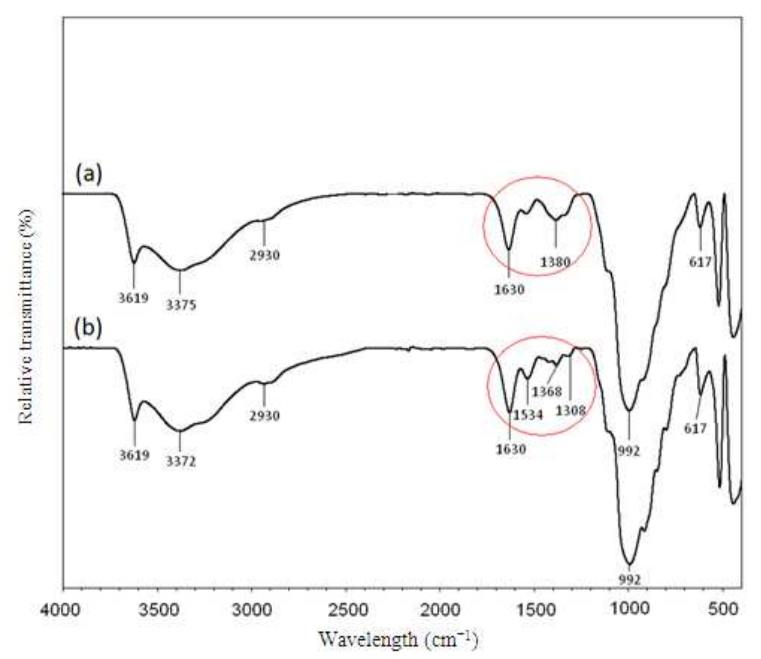

Fig. 6: FT-IR spectra of MMT/Cts and Ag/MMT/Cts BNCs (S5) (a) MMT/Cts/BNCs (S5) (b) $\mathrm{MMT} / \mathrm{Cts}$

The spectrum of Cts (Fig. 5a) shows peaks at $3292 \mathrm{~cm}^{-1}$ due to the overlapping of $\mathrm{O}-\mathrm{H}$ and $\mathrm{N}-\mathrm{H}$ stretching bands, $2878 \mathrm{~cm}^{-1}$ for aliphatic C-H stretching, 1643 and $1578 \mathrm{~cm}^{-1}$ for N-H bending and 1416, 1376 and $1306 \mathrm{~cm}^{-1}$ for $\mathrm{C}-\mathrm{H}$ bending, $1012 \mathrm{~cm}^{-1}$ for $\mathrm{C}-\mathrm{O}$ stretching. The spectrum of the MMT/Cts (Fig. 5b) shows the combination of characteristic absorptions due to the MMT and amine groups of chitosan. The peaks of- $\mathrm{NH}_{2}$ groups in pure chitosan at 1643 and $1578 \mathrm{~cm}^{-1}$ are shifted to 1630 and $1534 \mathrm{~cm}^{-1}$ in the MMT/Cts, corresponding to the deformation vibration of amine group of chitosan ${ }^{[21]}$. These results are in agreement with the data from PXRD, revealing the intercalation of chitosan in the MMT structure. As shown in Fig. 6, there is no significant change in the spectra of MMT/Cts and Ag/MMT/Cts BNCs, except for the disappearance of the peak at 1368 and $1308 \mathrm{~cm}^{-1}$ for MMT/Cts and the existence of the peak at $1380 \mathrm{~cm}^{-1}$ due to the complexation between the amino groups of the chitosan and Ag-NPs.

\section{CONCLUSION}

The silver nanoparticles were successfully prepared in the interlamellar space of MMT by using UV-irradiation, without any reducing agent or heat treatment. The Ag-NPs were found to be stabilized by chitosan molecules. The size distributions of Ag-NPs prepared at different UV-irradiation times indicated that larger Ag-NPs were obtained when irradiated for $3 \mathrm{~h}$. Further experiments showed that increase in UVirradiation time caused decrease in particles size and their size distributions. The synthesized Ag/MMT/Cts BNCs are stable in aqueous solutions and have potential applications as antimicrobial products.

\section{REFERENCES}

1. Kumar, M.N.V.R., 2002. A review of Chitin and Chitosan applications. React. Funct. Polym., 46: 1-27. DOI: 10.1016/S1381-5148(00)00038-9

2. Dufresne, A., J.Y. Cavaille, D. Dupeye, M.G. Ramirez and J. Romero, 1999. Morphology, phase continuity and mechanical behaviour of polyamide 6/chitosan blends. Polymer, 40: 1657-1666. DOI: 10.1016/S0032-3861(98)00335-8

3. Fuentes, S., P.J. Retuert, A. Ubilla, J. Fernandez and G. Onzalez, 2000. Relationship between composition and structure in Chitosan-based hybrid films. Biomacromolecules, 1: 239-243. DOI: 10.1021/BM0055091

4. Tanabe, T., N. Okitsu, A. Tachibana and K. Yamauchi, 2002. Preparation and characterization of keratinchitosan composite film. Biomaterials, 23: 817-825. DOI: 10.1016/S0142-9612(01)00187-9

5. Belova, V., H. Möhwald and D.G. Shchukin, 2008. Sonochemical intercalation of preformed gold nanoparticles into multilayered clays. Langmuir, 24: 9747-9753. DOI: 10.1021/LA8010822 
6. Paek, S.M., J.U. Jang, S.J. Hwang and J.H. Choy, 2006: Exfoliation-restacking route to $\mathrm{Au}$ nanoparticle-clay nanohybrids. J. Phys. Chem. Solids, 67: 1020-1023. DOI: 10.1016/J.JPCS.2006.01.021

7. Kozak, M. and L.J. Domka, 2004. Adsorption of the quaternary ammonium salts on montmorillonite. J. Phys. Chem. Solids, 65: 441-445. DOI: 10.1016/J.JPCS.2003.09.015

8. Twu, Y.K., Y.W. Chen and C.M. Shih, 2008. Preparation of silver nanoparticles using chitosan suspensions. Powder Technol., 185: 251-257. DOI: 10.1016/J.POWTEC.2007.10.025

9. Darroudi, M., M.B. Ahmad, K. Shameli, A.H. Abdullah and N.A. Ibrahim, 2009. Synthesis and characterization of UV-irradiated silver/montmorillonite nanocomposites. Solid State Sci., 11: 1621-1624. DOI: 10.1016/J.SOLIDSTATESCIENCES.2009.06.016

10. Yin, H., T. Yamamoto, Y. Wada and S. Yanagida, 2004. Large-scale and size-controlled synthesis of silver nanoparticles under microwave irradiation. Mater. Chem. Phys., 83: 66-70. DOI: 10.1016/J.MATCHEMPHYS.2003.09.006

11. Khanna, P.K., N. Singh, S. Charan, V.V.V.S. Subbarao, R. Gokhale and U.P. Mulik, 2005. Synthesis and characterization of Ag/PVA nanocomposite by chemical reduction method. Mater. Chem. Phys., 93: $117-121$.

DOI: 10.1016/J.MATCHEMPHYS.2005.02.029

12. Chen, P., L. Song, Y. Liu and Y. Fang, 2007. Synthesis of silver nanoparticles by [gamma]-ray irradiation in acetic water solution containing chitosan. Radiat. Phys. Chem., 76: 1165-1168. DOI: 10.1016/J.RADPHYSCHEM.2006.11.012

13. Huang, H. and Y. Yang, 2008. Preparation of silver nanoparticles in inorganic clay suspensions. Compos. Sci. Technol., 68: 2948-2953. DOI: 10.1016/J.COMPSCITECH.2007.10.003

14. Kamat, P.V., M. Flumiani and G.V. Hartland, 1998. Picosecond dynamics of silver nanoclusters. Photoejection of electrons and fragmentation. J. Phys. Chem. B., 102: 3123-3128. DOI: 10.1021/JP980009B
15. Patakfalvi, R., A. Oszko and I. Dékány, 2003. Synthesis and characterization of silver nanoparticle/kaolinite composites. Colloid Surface A., 220: 45-54. DOI: 10.1016/S09277757(03)00056-6

16. Temgire, M.K. and S.S. Joshi, 2004. Optical and structural studies of silver nanoparticles. Radiat. Phys. Chem., 71: 1039-1044. DOI: 10.1016/J.RADPHYSCHEM.2003.10.016

17. Prasad, V., C.D. Souza, D. Yadav, A.J. Shaikh and N. Vigneshwaran, 2006. Spectroscopic characterization of zinc oxide nanorods synthesized by solid-state reaction. Spectrochim. Acta A., 65: 173-178. DOI: 10.1016/J.SAA.2005.10.001

18. Bohren, C.F. and D.R. Huffman, 1998. Absorption and Scattering of Light by Small Particles. John Wiley-VCH Verlag, ISBN: 978-0-471-29340-8, pp: 544.

19. Liu, Z., H. Wang, H. Li and X. Wang, 1998. Red shift of plasmon resonance frequency due to the interacting $\mathrm{Ag}$ nanoparticles embedded in single crystal $\mathrm{SiO}_{2}$ by implantation. Applied Phys. Lett., 72: 1823-1825. DOI: 10.1063/1.121196

20. Alemdar, A., N. Güngör, Ö.I. Ece and O. Atici, 2005. The rheological properties and characterization of bentonite dispersions in the presence of non-ionic polymer PEG. J. Mater. Sci., 40: 171-177. DOI: 10.1007/S10853-005-5703-4

21. Ebru, G., D. Pestreli, C.H. Ünlü, O. Atici and N. Güngör, 2007. Synthesis and characterization of chitosan-MMT biocomposite systems. Carbohyd. Polymer, 67: 358-365. DOI: 10.1016/J.CARBPOL.2006.06.004 